\title{
Supplement to \\ Peptide retention time prediction yields improved tandem mass spectrum identification for diverse chromatography conditions
}

\author{
Aaron A. Klammer* \\ Xianhua $\mathrm{Yi}^{*}$ \\ aklammer@u.washington.edu \\ xhyi@u.washington.edu \\ Michael J. MacCoss* \\ William Stafford Noble*† \\ maccoss@gs.washington.edu \\ noble@gs.washington.edu
}

May 8, 2007

\footnotetext{
${ }^{*}$ Department of Genome Sciences, University of Washington, Seattle, WA, USA

† Department of Computer Science, University of Washington, Seattle, WA, USA
} 

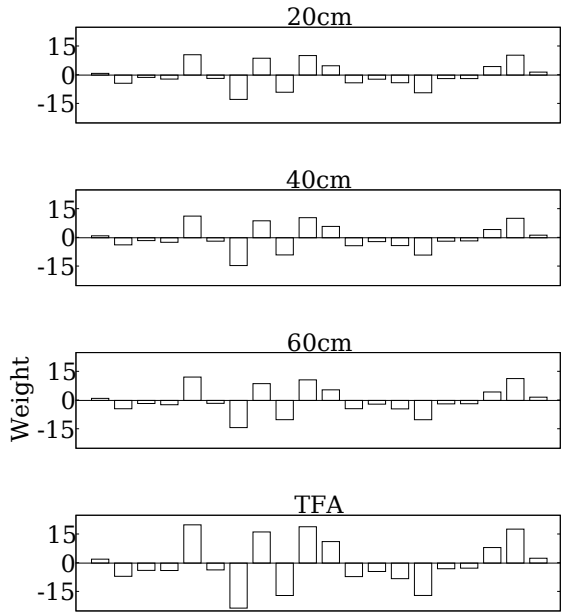

Chymotrypsin
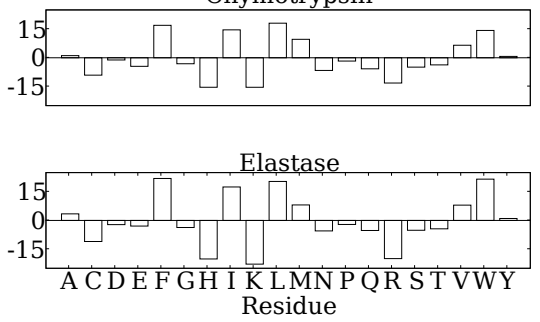
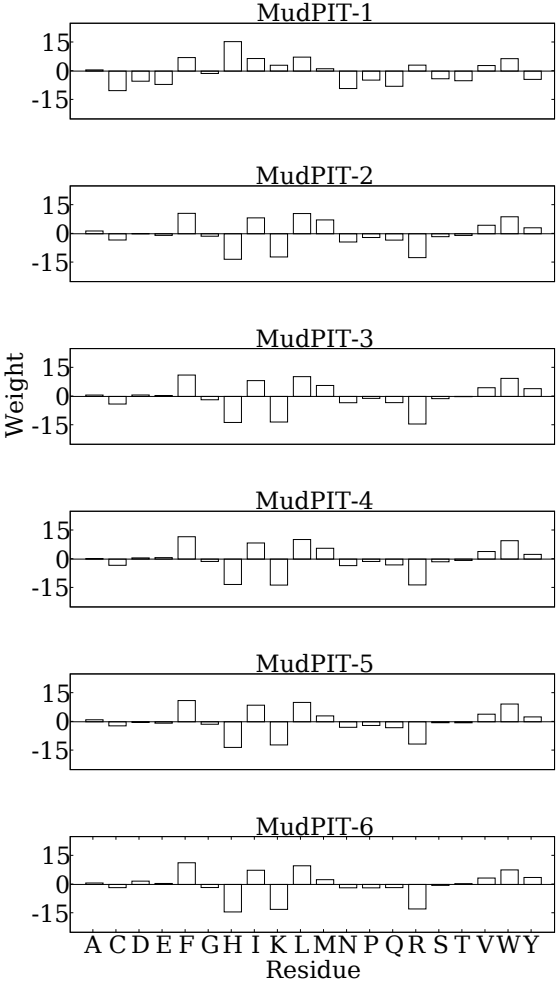

Figure 1: Contributions to retention time (RT). Shown are the support vector regression weights for the linear kernel for the 20 features corresponding to peptide amino acid composition; higher values indicate a positive contribution to RT. 


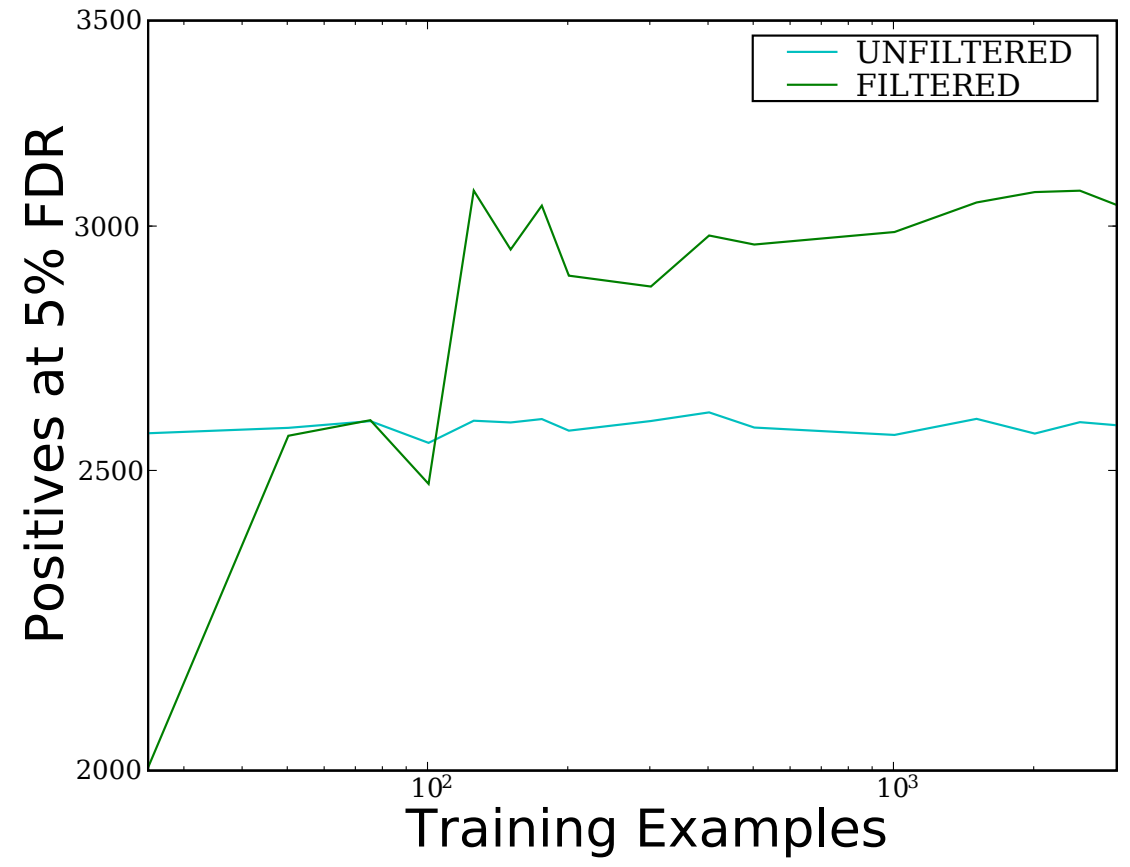

Figure 2: Filtering performance as a function of number of training examples. Shown are the number of true positives at 5\% FDR vs the number of peptides used to train the retention time support vector regressor for the TFA data set. 